\title{
Spin dynamics of ZnSe-ZnTe nanostructures grown by migration enhanced molecular beam epitaxy
}

Vasilios Deligiannakis, Siddharth Dhomkar, Haojie Ji, Daniela Pagliero, Igor L. Kuskovsky, Carlos A. Meriles, and Maria C. Tamargo

Citation: Journal of Applied Physics 121, 115702 (2017); doi: 10.1063/1.4978314

View online: https://doi.org/10.1063/1.4978314

View Table of Contents: http://aip.scitation.org/toc/jap/121/11

Published by the American Institute of Physics

\section{Articles you may be interested in}

Optical anisotropy in type-II ZnTe/ZnSe submonolayer quantum dots

Journal of Applied Physics 119, 224306 (2016); 10.1063/1.4953675

Molecular beam epitaxy of 2D-layered gallium selenide on GaN substrates

Journal of Applied Physics 121, 094302 (2017); 10.1063/1.4977697

Universal rules for visible-light absorption in hybrid perovskite materials

Journal of Applied Physics 121, 115501 (2017); 10.1063/1.4978071

An analysis of point defects induced by $\mathrm{In}, \mathrm{Al}, \mathrm{Ni}$, and $\mathrm{Sn}$ dopants in Bridgman-grown CdZnTe detectors and their influence on trapping of charge carriers

Journal of Applied Physics 121, 115701 (2017); 10.1063/1.4978377

Magnetic quantum dot in two-dimensional topological insulators

Journal of Applied Physics 121, 114302 (2017); 10.1063/1.4978632

Nonlinear UV absorption properties of bulk $4 \mathrm{H}-\mathrm{SiC}$

Journal of Applied Physics 121, 115703 (2017); 10.1063/1.4978768

\section{AIP |roumal af}

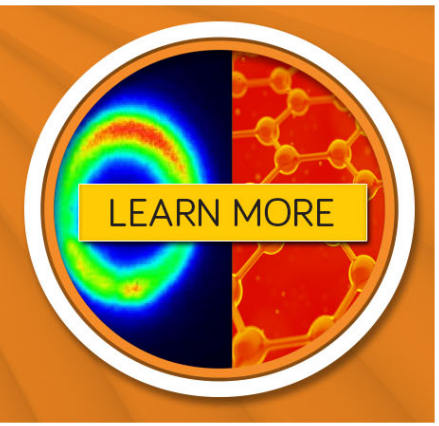




\title{
Spin dynamics of ZnSe-ZnTe nanostructures grown by migration enhanced molecular beam epitaxy
}

\author{
Vasilios Deligiannakis, ${ }^{1,2}$ Siddharth Dhomkar, ${ }^{3,2}$ Haojie Ji,, ${ }^{3,2}$ Daniela Pagliero, ${ }^{1}$ \\ Igor L. Kuskovsky, ${ }^{3,2}$ Carlos A. Meriles, ${ }^{1,2}$ and Maria C. Tamargo ${ }^{1,2}$ \\ ${ }^{1}$ City College of New York, CUNY, New York, New York 10031, USA \\ ${ }^{2}$ The Graduate Center of CUNY, New York, New York 10016, USA \\ ${ }^{3}$ Queens College, CUNY, Flushing, New York 11367, USA
}

(Received 22 November 2016; accepted 25 February 2017; published online 16 March 2017)

\begin{abstract}
We study the spin dynamics of ZnSe layers with embedded type-II ZnTe quantum dots using time resolved Kerr rotation (TRKR). Three samples were grown with an increasing amount of Te, which correlates with increased quantum dot (QD) density. Samples with a higher quantum dot density exhibit longer electron spin lifetimes, up to $\sim 1 \mathrm{~ns}$ at low temperatures. Tellurium isoelectronic centers, which form in the ZnSe spacer regions as a result of the growth conditions, were probed via spectrally dependent TRKR. Temperature dependent TRKR results show that samples with high QD density are not affected by an electron-hole exchange dephasing mechanism. Published by AIP Publishing.
\end{abstract}

[http://dx.doi.org/10.1063/1.4978314]

\section{INTRODUCTION}

Systems of reduced dimensionality, such as quantum wells (QWs), quantum dots (QDs), and defect centers have been proposed as promising candidates for spintronic applications. ${ }^{1} \mathrm{ZnSe}$ based semiconductors are particularly appealing and have also been recently explored for this purpose. ${ }^{2-4} \mathrm{In}$ contrast to their III-V counterparts (i.e., GaAs), ${ }^{5}$ II-VI based semiconductors have the advantage of having a low abundance of spin $1 / 2$ nuclear spins and reduced hyperfine couplings. Yet, spins in wide bandgap II-VI semiconductors have a strong electron-hole exchange interaction, which is the main dephasing mechanism at low temperatures, limiting lifetimes to tens of picoseconds due to fast hole-spin decoherence. ${ }^{6}$ Mino et al. $^{7}$ explored a type-II $\mathrm{ZnSe} / \mathrm{BeTe} \mathrm{QW}$ structure as a means to decouple the electrons and holes. Under photoexcitation electrons are localized in the ZnSe wells, and holes are in the BeTe barriers. They observed the longest spin dephasing times, as large as $6.1 \mathrm{~ns}$, for undoped QWs.

During the past several years, our group has investigated the growth of ultra-small type-II quantum dots of ZnTe embedded in a ZnSe matrix and explored their unique physical properties based on their type-II band alignment. ${ }^{8}$ In these structures the type-II band alignment ensures that holes are confined within the ZnTe-based QD regions, while electrons are delocalized in the $\mathrm{ZnSe}$ matrix. As a consequence of the growth mechanism, tellurium isoelectronic centers (TeICs) also form within the ZnSe matrix. In this paper, we report on the electron spin dynamics of these samples. Our results indicate that significantly longer electron spin lifetimes are achieved in the samples with larger densities of ZnTe QDs. We were also able to directly probe the spin dynamics of tellurium isoelectronic centers (Te-ICs) present in the $\mathrm{ZnSe}$ matrix, which have not been previously reported.

\section{GROWTH}

Three $\mathrm{ZnTe} / \mathrm{ZnSe}$ type-II QD nanostructure samples were studied having varying effective tellurium concentrations. The structures were grown by a combination of molecular-beam epitaxy (MBE) and migration enhanced epitaxy (MEE) on (001) oriented GaAs substrates. ${ }^{9}$ The structure for all the samples consists of a $55 \mathrm{~nm} \mathrm{ZnSe} \mathrm{buffer} \mathrm{layer,} \mathrm{followed} \mathrm{by} \mathrm{a}$ superlattice of 100 periods of $1.7 \mathrm{~nm} \mathrm{ZnSe}$ spacers and ZnTe QDs. The formation of ZnTe QDs was achieved by using a shutter sequence of alternating zinc and tellurium fluxes, known as MEE. ${ }^{9}$ Two different MEE shutter sequences were used to form the QDs. In the single cycle sequence, first the surface was exposed to a $\mathrm{Zn}$ flux only, followed by a short interruption, then the Te flux only was opened followed again by an interruption, and finally the $\mathrm{Zn}$ flux was opened before resuming the $\mathrm{ZnSe}$ spacer layer growth. Each step had a duration of $5 \mathrm{~s}$. The interruptions are intended to allow zinc and tellurium to aggregate, and form quantum dots. In the triple cycle, the alternating $\mathrm{Zn}$ and Te fluxes, separated by interruptions, were repeated three times before resuming the spacer layer growth. ${ }^{9}$

Sample A was grown with a single cycle sequence, while samples B and C were grown with triple cycle sequences. The Te flux was also varied to adjust the Te content of the samples, which was measured using high resolution $\mathrm{x}-$ ray diffraction (HRXRD) and secondary ion-mass spectroscopy (SIMS) as described in Ref. 10. Table I summarizes some of the relevant growth parameters. From the table, it is evident that sample A has the lowest average effective Te content, while sample $\mathrm{C}$ has the highest.

\section{EXPERIMENTAL MEASUREMENTS}

The samples were characterized using low temperature photoluminescence (PL) and time-resolved Kerr rotation (TRKR) measurements. TRKR measures the spin dephasing time of electrons and holes initiated by a circularly polarized pump light. A linearly polarized probe beam is focused at the same spot on the surface as the circularly polarized pump, and the polarization rotation in the probe beam induced by spin polarized carriers is measured as a function 
TABLE I. Important growth parameters for the three ZnTe/ZnSe QD samples.

\begin{tabular}{lccccc}
\hline \hline Sample & $\begin{array}{c}\text { Te Flux } \\
\left(10^{-7} \text { Torr }\right)\end{array}$ & $\begin{array}{c}\text { No. of MEE } \\
\text { cycle }\end{array}$ & No. of periods & $\%$ Te & $\%$ Strain \\
\hline A & 0.4 & 1 & 100 & 0.1 & 80 \\
B & 0.26 & 3 & 100 & 0.2 & 80 \\
C & 0.4 & 3 & 100 & 0.3 & 80 \\
\hline \hline
\end{tabular}

of the pump-probe time delay. Pulses of light are generated by a tunable mode-locked Ti:sapphire laser with a pulse width of $130 \mathrm{fs}$ and a repetition rate of $76 \mathrm{MHz}$. A degenerate pump probe setup is used. The area illuminated on the sample had a diameter of $\sim 100 \mu \mathrm{m}$. Typical excitation powers were 5 and $1 \mathrm{~mW}$. A coupled photodiode bridge was used to enhance the sensitivity of the probe signal. ${ }^{11} \mathrm{~A}$ photoelastic modulator was used to modulate the pump amplitude at $50 \mathrm{kHz}$, whereas the probe pulse was chopped mechanically at $5 \mathrm{kHz}$. Lock-in detection frequency difference allowed us to separate the pump and probe signals. Permanent magnets were attached to the sample holder of an optical cryostat to give a $\sim 0.5 \mathrm{~T}$ magnetic field perpendicular to the growth direction of the sample. PL measurements were performed with a TriVista SP2 500i Triple monochromator coupled with a thermoelectrically cooled CCD camera. Samples were loaded onto a closed system He-cryostat and cooled down to $8 \mathrm{~K}$. The 351-nm laser line from an Argon ion laser was used to excite samples with varying power from $0.06 \mathrm{~W} / \mathrm{cm}^{2}$ to $60 \mathrm{~W} / \mathrm{cm}^{2}$.

\section{RESULTS AND DISCUSSION}

Figure 1 shows the PL spectra at $8 \mathrm{~K}$, for the three samples studied as well as that of a reference bulk ZnSe sample $(100 \mathrm{~nm})$ grown in the same chamber. A single sharp nearband-edge peak is observed at $\sim 2.80 \mathrm{eV}$ in the $\mathrm{ZnSe}$ sample. By contrast, two prominent bands with maxima centered around $2.5 \mathrm{eV}$ (green band) and $2.7 \mathrm{eV}$ (blue band) can be identified in the three QD samples studied. Sample A, with the lowest Te concentration, is dominated by the highenergy blue band, which is overlaid with near-band-edge sharp lines originating from Te isoelectronic centers (ICs). The blue band has been identified to be due to tellurium clusters of $\mathrm{Te}_{\mathrm{n}>2}$ that form at anionic sites in the ZnSe spacer. ${ }^{9}$ A series of sharp lines can be resolved at $2.752 \mathrm{eV}, 2.759 \mathrm{eV}$, and $2.767 \mathrm{eV}$ that have been assigned to the non-nearest neighbors of Te pairs. ${ }^{9}$ Strong phonon coupling can be seen in all samples, with up to four phonon replicas separated by $32 \mathrm{meV}$ clearly resolved within the blue band. The green band in sample A is only evident by the presence of the long tail on the low energy side of the peak. Sample B shows a more well-defined green band but the blue band still dominates. Sharp lines are no longer resolved in the blue band but rather a peak at $2.759 \mathrm{eV}$ can be seen, which is identified as the combination of different transitions from several centers due to a larger distribution of different orientations of Te-IC pairs. Sample C, with the highest Te concentration, shows a dominant green band. This band has been previously
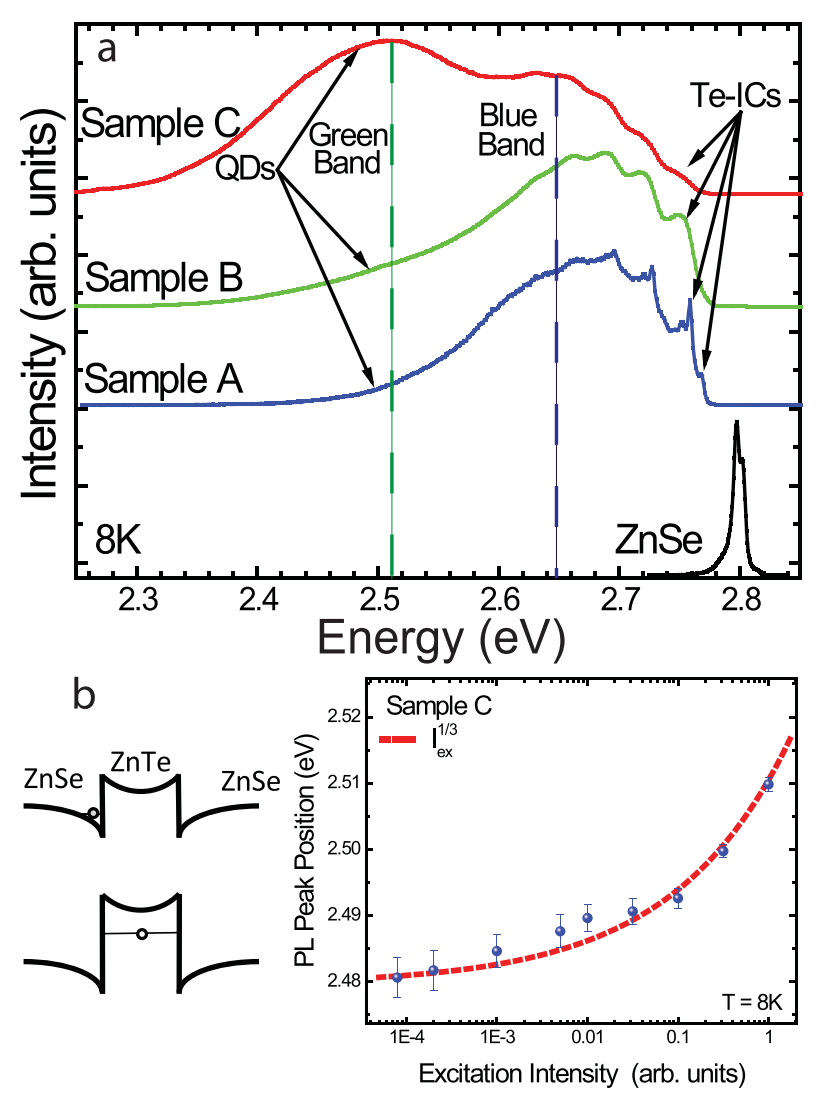

FIG. 1. (a) PL spectra for the three samples studied; a $\mathrm{ZnSe}$ bulk layer is also included for reference. The dashed lines at 2.51 and $2.70 \mathrm{eV}$ indicate the center of the green and blue bands, respectively. (b) Left: Type II band alignment of the ZnTe QDs in a ZnSe matrix illustrating the separation of carries, which leads to the observed intensity dependence of the peak position. Right: Excitation intensity dependence of the green band peak energy for sample $\mathrm{C}$. The dotted red line is a fit to a $1 / 3$ power dependence, consistent with type-II behavior.

identified with the type-II emission from ZnTe QDs and larger clusters of Te atoms. ${ }^{10}$ It has been shown that higher effective concentrations of Te in these type-II QD samples increase the green band intensity corresponding to a larger QD density. Excitation intensity dependent PL of the peak energy of the green band in sample $\mathrm{C}$ exhibits a one third power law dependence confirming the type-II band alignment between the ZnTe-based QDs and the ZnSe matrix. ${ }^{12}$ This is shown in Figure 1(b), along with a schematic of the type II band alignment.

A typical TRKR trace for sample $\mathrm{C}$ measured with and without an applied magnetic field is shown in Figure 2. Both pump and probe energies were tuned to $2.798 \mathrm{eV}$ near the bandedge of $\mathrm{ZnSe}$ at $80 \mathrm{~K}$. We observe that the Kerr rotation (KR) signal has an initial fast decay but is non-zero even at negative time delays at zero field, indicative of spins persisting from the previous laser pulse (13 ns). This is demonstrated by blocking the pump prior to the start of the measurement, allowing it to come through at some negative time delay, and then blocking it again, by which we clearly see a change in the KR signal. These long spin lifetimes outlast the ZnSe exciton lifetime typically observed to be 100 ps at low temperatures. ${ }^{13}$ One possible explanation for this is that the signal persists from resident electrons in the conduction band due to 


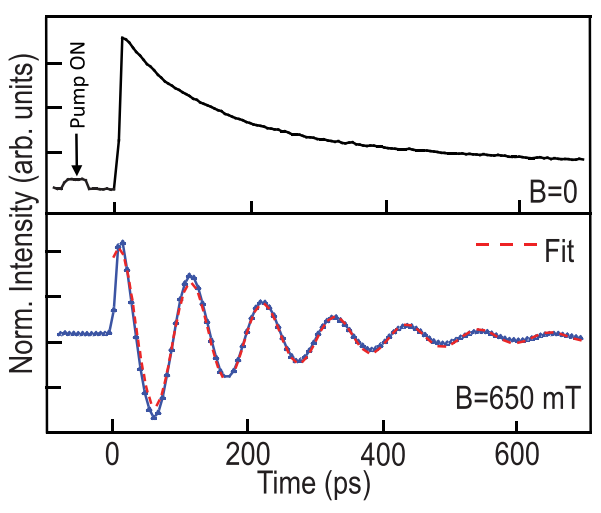

FIG. 2. Kerr rotation signal for sample $\mathrm{C}$ at $\mathrm{T}=75 \mathrm{~K}$ with (lower trace) and without (upper trace) an applied magnetic field in the Voigt geometry. The persistence of the signal at negative times is evident by the small signal in the zero field measurement when the pump is on during a short interval prior to the start of the experiment. The red line in the lower trace is a fit to the Larmor precession as discussed in the text.

the presence of the type-II QDs which form indirect excitons. These indirect excitons can last up to $86 \mathrm{~ns} .^{12}$ Quantum beat oscillations are observed when a magnetic field is applied in the Voigt geometry. The oscillations correspond to a Larmor precession through which the electron spin evolves. Oriented electron spins precess around a perpendicular component of the applied field. These oscillations can be fitted to the equation: $A \cos \left(g \mu_{B} B \Delta t / h\right)$, where $\mathrm{A}$ is the amplitude, $\mathrm{g}$ the effective g-factor of the sample, $\mu_{\mathrm{B}}$ the Bohr magneton, and $\mathrm{B}$ the magnitude of the total magnetic field experienced by the electrons. Using the above formula, we can fit the data to extract the electron g-factor. A $g$-factor of $\sim 1.1$ was found for all cases, very close in value to the electron $g$-factor in $\mathrm{ZnSe} .{ }^{14}$ This supports the notion that the electrons are within the $\mathrm{ZnSe}$ matrix. The fast dephasing time of 217 ps found with an applied magnetic field can be attributed to inhomogeneities within the ZnSe matrix from QDs and defects.

Figure 3 compares the TRKR contour plots for the three samples studied, measured at $\sim 10 \mathrm{~K}$. Pump and probe energies were kept degenerate, scanned over a region from $2.75 \mathrm{eV}$ to $2.82 \mathrm{eV}$ and plotted as a function of excitation energy. In sample A, shown in Figure 3(a), the TRKR signal vanishes for excitation energies below $2.785 \mathrm{eV}$. Pumping directly at energies corresponding to the Te-IC centers (blue band) and QDs (green band) did not show any TRKR signal. Keeping the pump helicity fixed, a spin flip is observed going from the $\mathrm{ZnSe}$ heavy hole $(\mathrm{HH})$ to light hole $(\mathrm{LH})$ excitation energies, indicated by the Kerr rotation signal changing from positive to negative at $2.795 \mathrm{eV}$ and $2.810 \mathrm{eV}$, respectively. The biaxial strain induced by the GaAs substrate splits the LH and HH degeneracy at the valence band minimum. The optical selection rules are such that when a LH is excited by a $\sigma$ - photon $(S=-1)$, a spin-down electron $\left(m_{\mathrm{s}}=-1 / 2\right)$ and a spin-up hole $\left(m_{\mathrm{s}}=1 / 2\right)$ are created simultaneously. The LH state relaxes quickly and is depolarized, whereas accumulation of spin-up electrons breaks the balance of spin population in the conduction band. ${ }^{15}$ This is observed as a negative polarization by the linearly polarized probe. The spin lifetimes observed in this sample were very short, on the order of tens of picoseconds. Similar TRKR lifetimes were measured by us (not shown) and have been observed by others ${ }^{15}$ for bulk undoped $\mathrm{ZnSe}$ at low temperatures.

Samples B and C, with a larger amount of Te $(0.2 \%$ and $0.3 \%$, respectively), behave quite differently from sample A (which has $0.1 \%$ Te content). The data are shown in Figures 3(b) and 3(c). In both samples the TRKR signals persist as we probe deeper in energy into the region of the blue band, which originates from the Te-ICs. We interpret these observations by considering that we are able to probe the Te ICs in these samples that have increased Te content. Direct excitation of Te-bound excitons has been observed as a phonon-broadened peak below the band edge in photoluminescence excitation spectroscopy experiments in other II-VI materials. ${ }^{16}$ In particular, in sample $\mathrm{C}$ we are able to probe deep within the bandedge of $\mathrm{ZnSe}$ at energies that clearly correspond to Te ICs. Sample C exhibits long spin lifetimes of up to $1 \mathrm{~ns}$ at low temperatures, in sharp contrast to the lifetimes measured in

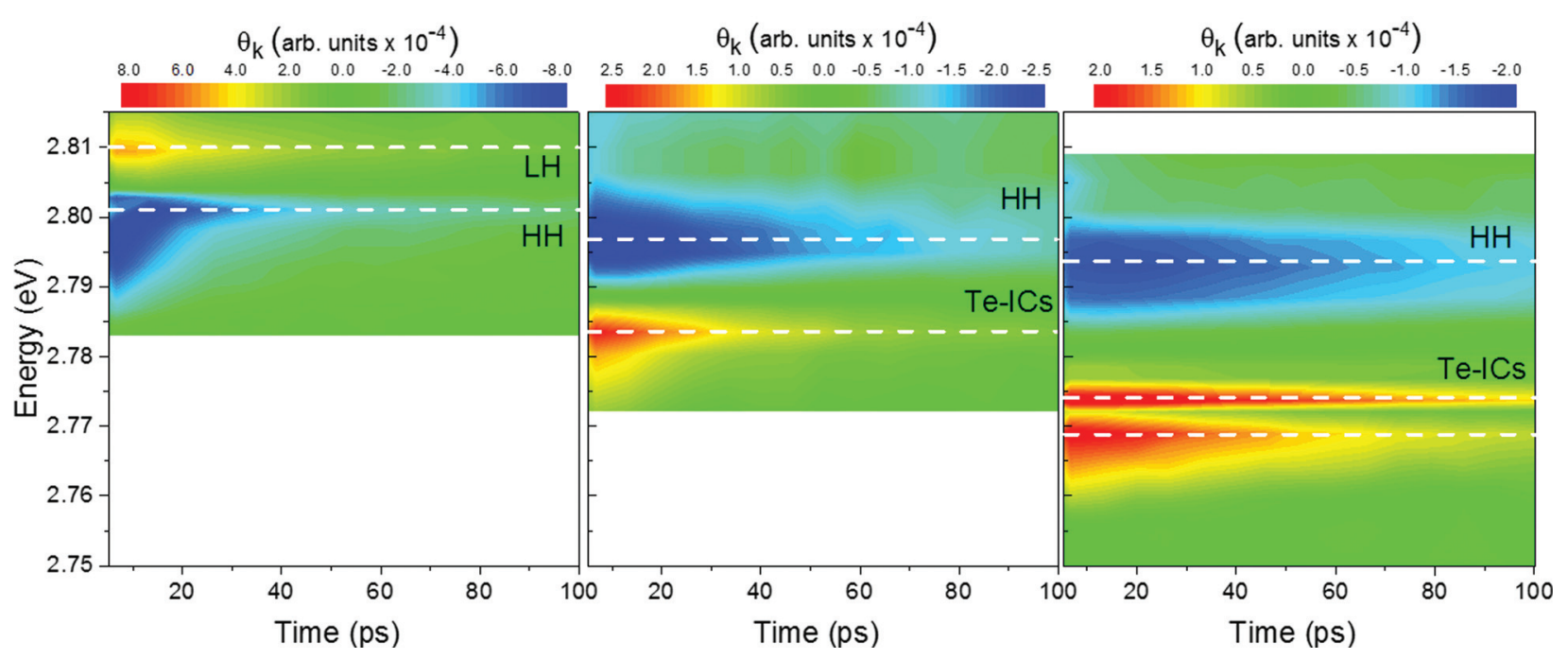

FIG. 3. Spectral dependence of the TRKR signal for samples A through C. Dashed lines indicate the position of the excitonic levels associated with the ZnSe spacer layers. Energies corresponding to direct excitation of Te-ICs are also labeled. Regions with no color indicate the absence of a TRKR signal. 
sample A. In addition, we observe a spin flip near the energies corresponding to the $\mathrm{Te}$ isoelectronic centers for both samples $\mathrm{C}$ and $\mathrm{B}$. The origin of this spin flip is still under investigation. Probing at energies around the LH to free exciton (FE) transition of $\mathrm{ZnSe}$ the TRKR signal is significantly diminished. In addition, we do not observe the spin flip seen in bulk $\mathrm{ZnSe}$ and in sample A due to the non-degenerate $\mathrm{LH}$ and $\mathrm{HH}$. We tentatively explain the latter behavior for samples B and C as being due to the capture of light holes by the presence of the type-II QDs within the samples. These ZnTe-based QDs form a staggered band alignment with the $\mathrm{ZnSe}$ matrix producing a potential well for holes (see Figure 1(b)). Moreover, trying to probe the green band in all samples did not produce a TRKR signal. This was not unexpected since the type-II alignment of the QDs results in optically forbidden transitions.

To better characterize the increase in spin lifetime observed for the samples with higher Te content, we investigated the temperature dependence of the Te-IC center related spin lifetimes in samples A and C. Sample B, with an intermediate $\mathrm{Te}$ content, was also measured. Here we compare $\mathrm{A}$ and $\mathrm{C}$ since they exhibit the largest effect. In order to probe the Te-IC centers directly we used the lowest energies at which we observed the TRKR signal for each sample. This value varied depending on the Te content of each sample. For sample A, this was near the band edge of $\mathrm{ZnSe}$, at $2.81 \mathrm{eV}$ and for sample $\mathrm{C}$ it was at $2.75 \mathrm{eV}$. Figure 4 shows the temperature dependence of the TRKR lifetime for samples $\mathrm{A}$ and $\mathrm{C}$. In both cases, the TRKR measurements typically exhibit a bi-exponential decay which is denoted by a long $\tau_{2}^{*}$ and a short $\tau_{2}^{*}$ on the graphs. This bi-exponential decay is illustrated in a typical TRKR decay curve for sample C, shown as an inset in Figure 4. The fast component of the initial decay can be explained by the rapid decoherence of the hole spins initialized by the pump, while the long decay corresponds to the electron spin decoherence lifetime.

The principal observation is that sample $\mathrm{C}$ exhibits a much longer long $\tau^{*}{ }_{2}$ lifetime at low temperature, exceeding $1 \mathrm{~ns}$. The value of the long $\tau^{*}{ }_{2}$ increases further as the temperature grows but an exact determination of its value is limited by the delay stage (the maximum displacement corresponds to $1 \mathrm{~ns})$. For sample A the value of the long $\tau^{*}{ }_{2}$ is much shorter below $50 \mathrm{~K}$, resulting in a single exponential decay behavior. Above $40 \mathrm{~K}$ the long $\tau_{2}{ }_{2}$ spin lifetime measured is $\sim 100 \mathrm{ps}$. This behavior is similar to that seen in bulk undoped $\mathrm{ZnSe}$ (not shown). This sharp drop off of the lifetime at $\sim 50 \mathrm{~K}$ has been explained as the motional narrowing of excitons. ${ }^{17} \mathrm{In}$ that mechanism, electron and hole overlap increases at lower temperatures. Since hole spin decoherence is much faster, it contributes to the decrease of the electron spin lifetime, lasting only tens of picoseconds. In sample $\mathrm{C}$, which has a much larger density of type-II quantum dots, this decoherence mechanism does not seem to play a dominant role. A similar behavior to that of sample $\mathrm{C}$ has been observed in undoped type-II quantum wells, which also exhibit spin lifetimes in excess of $1 \mathrm{~ns}$ at low temperatures. ${ }^{7}$ The decoherence mechanism responsible for the behavior of sample $\mathrm{C}$ has not been established.

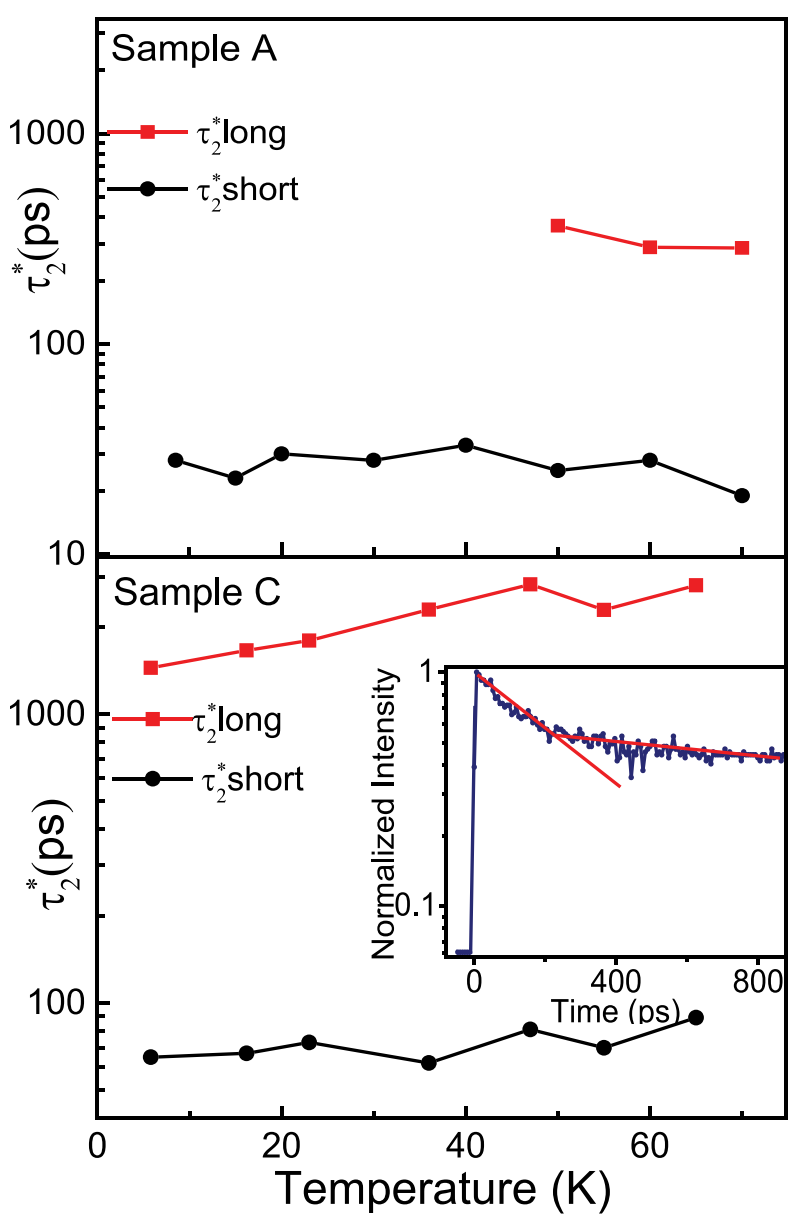

FIG. 4. Temperature dependent spin relaxation lifetimes measured by TRKR pumped and probed at 2.78 and $2.75 \mathrm{eV}$ for samples A and C, respectively. Typically a biexponential decay is observed consisting of a long and short lifetime component illustrated in the TRKR shown in the inset for sample $\mathrm{C}$ at $65 \mathrm{~K}$. Red lines in the inset indicate straight lines for the two separate decays observed in the curve.

\section{CONCLUSION}

In conclusion, we have investigated the spin decoherence lifetimes of ZnSe samples with embedded ZnTe type-II QDs. Three samples with different effective Te content, $0.1 \%, 0.2 \%$, and $0.3 \%$, were investigated using spectrally resolved TRKR. Low temperature PL confirms the presence of type II QDs which increase in density with increasing Te- content, as well as with the presence of Te-ICs in the $\mathrm{ZnSe}$ barrier regions. We found that the QDs could not be probed directly by TRKR, which we attribute to their type-II nature. On the other hand, the TRKR signal from the Te ICs that are present in the $\mathrm{ZnSe}$ barrier regions could be observed, but only in the samples with a higher Te content, and consequently with larger QD densities. The spin coherence lifetimes increase significantly as the Te content increases in the samples. The sample with the highest Te content exhibited a lifetime of $\sim 1$ ns at $10 \mathrm{~K}$, compared to less than $20 \mathrm{ps}$ for the sample with the lowest Te content. Furthermore, a spin flip near the energy of the Te-IC could be detected in the samples with high Te content. The spin flip near the band-edge seen in pure $\mathrm{ZnSe}$ was not observed in samples with large Te content, which hints at the capture of light holes by the type-II QDs present in the samples. 


\section{ACKNOWLEDGMENTS}

This research was supported by the U.S. Department of Energy, Office of Basic Energy Sciences, Division of Materials Sciences and Engineering under Award No. SC003739.

One of us (M.C.T.) also acknowledges the support from the NSF CREST Center IDEALS (HRD-1547830).

${ }^{1}$ Spin Physics in Semiconductors, edited by M. I. Dyakonov (Springer, 2008).

${ }^{2}$ E. A. Zhukov, D. R. Yakovlev, A. Schwan, O. A. Yugov, A. Waag, L. W. Molenkamp, and M. Bayer, Phys. Status Solidi B 251, 1872 (2014).

${ }^{3}$ A. Greilich, A. Pawlis, F. Liu, O. A. Yugov, D. R. Yakovlev, K. Lischka, Y. Amamoto, and M. Bayer, Phys. Rev. B 85, 121303 (2012).

${ }^{4}$ I. Malajovich, J. M. Kikkawa, D. D. Awschalom, J. J. Berry, and N. Samarth, J. Appl. Phys. 87, 5073 (2000).

${ }^{5}$ D. J. Sleiter, K. Sanaka, Y. M. Kim, K. Lischka, A. Pawlis, and Y. Yamamoto, Nano Lett. 13, 116 (2013).

${ }^{6}$ J. He, H. Zhong, and G. D. Scholes, Phys. Rev. Lett. 105, 046601 (2010).

${ }^{7}$ H. Mino, Y. Kouno, K. Oto, K. Muro, R. Akimoto, and S. Takeyama, Appl. Phys. Lett. 92, 153101 (2008).
${ }^{8}$ B. Roy, H. Ji, S. Dhomkar, F. J. Cadieu, L. Peng, R. Moug, M. C. Tamargo, Y. Kim, D. Smirnov, and I. L. Kuskovsky, Phys. Rev. B 86, 165310 (2012).

${ }^{9}$ I. L. Kuskovsky, C. Tian, G. F. Neumark, J. E. Spanier, I. P. Herman, S. P. Guo, and M. C. Tamargo, Phys. Rev. B 63, 155205 (2001).

${ }^{10} \mathrm{~S}$. Dhomkar, H. Ji, B. Roy, V. Deligiannakis, A. Wang, M. C. Tamargo, and I. L. Kuskovsky, J. Cryst. Growth 422, 8 (2015).

${ }^{11}$ S. A. Crooker, D. D. Awschalom, and N. Samarth, IEEE J. Sel. Top. Quantum Electron. 1, 1082 (1995).

${ }^{12}$ Y. Gu, I. L. Kuskovsky, M. van der Voort, G. F. Neumark, X. Zhou, and M. C. Tamargo, Phys. Rev. B 71, 045340 (2005).

${ }^{13}$ I. S. Hauksson, J. Suda, Y. Kawakami, Sz. Fujita, and Sg. Fujita, J. Cryst. Growth 159, 329 (1996).

${ }^{14}$ S. Ghosh, N. P. Stern, B. Maertz, D. D. Awschalom, G. Xiang, M. Zhu, and N. Samarth, Appl. Phys. Lett. 89, 242116 (2006).

${ }^{15}$ J. M. Kikkawa, I. P. Smorchkova, N. Samarth, and D. D. Awschalom, Science 277, 1284 (1997).

${ }^{16}$ K. Dhese, J. Goodwin, W. E. Hagston, J. E. Nicholls, J. J. Davies, B. Cockayne, and P. J. Wright, Semicond. Sci. Technol. 7, 1210 (1992).

${ }^{17}$ M. Z. Maialle, E. A. de Andrada e Silva, and L. J. Sham, Phys. Rev B 47, 15776 (1993). 